\title{
Application of the most probable number method to estimations of entomopathogenic nematode populations in soil
}

\author{
By B HASS, C T GRIFFIN, J G KEATING* and M J DOWNES \\ Department of Biology, National University of Ireland, Maynooth, \\ Co. Kildare, Ireland
}

(Accepted 31 October 1997)

\begin{abstract}
Summary
The calculation of most probable numbers (mpn) was used for the estimation of numbers of infective entomopathogenic nematodes in soil. The mpn concept was first introduced in bacteriology as a means of estimating numbers of organisms in a substrate without a direct count, in cases where direct enumeration could not be applied. In the work reported here, soil samples infested with infective juveniles (IJs) of Heterorhabditis megidis (isolate HF85) were diluted with uninfested soil and the diluted soils were baited with mealworm larvae (Tenebrio molitor). The mpn of infective units of IJs in the undiluted soil was calculated. The mpn calculation was found to be applicable to entomopathogenic nematodes in soil under particular conditions. It could be successfully applied to data for the response of soil units but not to the data based on the response of individual insects, as the latter did not confirm to a Poisson series. The calculated mpn represented between 2.9 and $7.1 \%$ of the initial inoculum of IJs. It was suggested that IJs might act as a group in infecting an insect host. Using the data for Tenebrio mortality on parasitisation, the mpn based on quantal response of the mealworms would therefore not give the true density of IJs in the soil sample but the effective density, or the quantity of infective units. Although the biological significance of the infective unit needs further clarification, mpn was found to be a useful parameter for use in comparative experiments.
\end{abstract}

Key words: Most probable number, infectivity, infective unit, dilution method, baiting technique, entomopathogenic nematodes, Heterorhabditis

\section{Introduction}

Entomopathogenic nematodes of the genera Steinernema and Heterorhabditis are obligate parasites of a wide range of insects. Together with their mutualistic bacteria Xenorhabdus and Photorhabdus (respectively), they cause insect death within days. The nematodes develop and reproduce inside the insect cadaver from which infective juveniles (IJs) are released into the soil, where they persist without feeding and seek out new hosts (Poinar, 1975).

Persistence of entomopathogenic nematodes in soil can be monitored in several different ways. One possible approach is to bait the soil samples and to use the mortality of insect hosts

*Department of Computer Science, National University of Ireland, Maynooth, Kildare, Ireland 
(e.g. mealworms) as indicators of soil infectivity (Koppenhöfer, Kaya \& Taormino, 1995). A drawback of this type of baiting technique is the lack of sensitivity in response when infectivity is high such that $100 \%$ of insects are killed. In such cases, dissection of hosts can provide data on the number of nematodes that have invaded (Fan \& Hominick, 1991), but such dissections are labour intensive. Measures of infectivity can be gained from insect mortality, no matter how highly infective the sample, by the use of dilution methods. In the work reported here, a novel soil dilution technique was used by which nematode infested soil samples were diluted with uninfested soil and the diluted soils were baited. Using this technique, an LD50 or the 'dose' of undiluted soil required to kill $50 \%$ of the bait insects could be calculated by probit analysis (Hass, 1996). An alternative statistical treatment of data resulting from baiting a soil dilution series is the calculation of the most probable number (mpn) of infective units in the undiluted soil. The mpn concept goes back to McCrady (1915) and has been described and discussed in detail by Halvorson \& Ziegler $(1933 a, b)$. It was introduced as a means of estimating numbers of organisms in a liquid without any direct count, in cases where direct enumeration could not be applied, as summarised in Gerhardt $e t$ al. (1981). The mpn method has been used for the estimation of bacterial numbers in liquids (e.g. Cochran, 1950; Taylor, 1962) and in soil (Rowe, Todd \& Waide, 1977), for infective units of a plant parasitic fungus (Downes, 1972) and for estimates of rhizobia in soil (e.g. Woomer, Singleton \& Bohlool, 1988; Turk \& Keyser, 1993). It has not previously been used for the estimation of entomopathogenic nematode numbers in soil.

The mpn technique is based on probability theory and relies on the determination of the presence or absence of the organism of interest in each of several consecutive dilutions of several replicate dilution series prepared from the sample being tested. The density of organisms in the original fluid is calculated from the observed number of dilutions that show a positive reaction (e.g. growth of the organism) when samples from them are incubated in suitable media. Two principal assumptions underly this technique. The first is that the organisms are randomly distributed in the fluid during dilution and sampling. The second is that each incubated sample exhibits growth in the culture medium whenever the sample contains one organism or more. The concentration of effective organisms (e.g. bacteria that can grow) is estimated in two steps: [1] Testing whether the results of the dilution series conform to a Poisson series by using Moran's test (Moran 1954a,b) or Stevens' test (Stevens, 1958) and, if so, [2] obtaining the most probable estimate of the count. This estimate can be obtained either from published tables, given for different dilution ratios and degrees of replication in Jones (1979), or by computation. For over-coming the inflexibility of mpn procedures computer programs generating individual mpn values (Koch, 1982; Russek \& Colwell. 1983: Klee, 1993) have been developed. Moran (1954a) suggests a test for match to the Poisson series: this test can only be applied when, at the extremes of the range of dilutions used, $100 \%$ positive and $100 \%$ negative scores are obtained.

The application of the mpn method to entomopathogenic nematodes in soil is novel. In this paper we aim to evaluate whether the estimation of most probable number of infective units represents a useful method, complementary to other methods for detecting changes in infectivity of entomopathogenic nematodes in soil.

\section{Materials and Methods}

Soil dilution series were prepared by inoculating soil with infective juveniles (IJs) of Heterorhabditis megidis Poinar, Jackson \& Klein (isolate HF85) and diluting it with the same, but uninfested, soil. The most probable number of effective organisms (IJs that can infect) in 
soil samples was estimated using the insect mortality data obtained from baiting several replicate, two-fold, soil dilution series with Tenebrio molitor Linnaeus larvae (mealworms).

The soil for the two experiments reported here came from Maynooth College garden and had the following composition by weight: $14.7 \%$ clay, $37.4 \%$ silt, $25.4 \%$ sand with $22.5 \%$ organic matter. The proportion of sand, silt and clay was estimated by the Teagasc Soil Laboratory, Johnstown Castle, Ireland. The soil was air dried on the laboratory bench to a moisture content of 23\% (Expt 1) and 27\% (Expt 2) by weight. The soil used was unsterilised but Maynooth soil is naturally free of Heterorhabditis. Throughout this paper one unit of soil equals $120 \mathrm{~g}$.

Heterorhabditis megidis (isolate HF85) was obtained from P Westerman and W R Simons as an isolate from Flevoland, The Netherlands. The nematodes were reared in Tenebrio larvae at $20^{\circ} \mathrm{C}$ and IJs were harvested from modified White traps (White, 1927), washed by sedimentation and applied to the soil units on the same day. The numbers used in each experiment and the dilution series that were prepared are described below under the individual experiments.

The baiting method used for monitoring the infectivity of nematode infested soil samples was based on a method described by Bedding \& Akhurst (1975). Forty Tenebrio larvae were gently mixed into $120 \mathrm{~g}$ of test soil in a $250 \mathrm{ml}$ lidded plastic beaker and incubated for 4 days at $20^{\circ} \mathrm{C}$ in the dark. Afterwards, the Tenebrio larvae were removed from the soil and the numbers dead and alive were recorded. The live ones were stored for another 3 days at $20^{\circ} \mathrm{C}$ in petri dishes with some wheat bran flakes (Odlum Group Ltd, Dublin) before the final record of the proportion dead and parasitised by Heterorhabditis (as evidenced by characteristic colour) was taken. All soil mixing was carried out on a slowly rotating tumble mixer that accepted all replicates for any dilution level at the same time.

\section{Expt 1. Insect mortalities at different soil dilutions as a basis for mpn calculations}

Seven of 10 units (beakers) of soil were each inoculated with $2 \mathrm{ml}$ of an aqueous suspension of $16000 \mathrm{IJs} \mathrm{ml}^{-1}$. The other three served as untreated controls, receiving $2 \mathrm{ml}$ of tap water only. A two-fold dilution series was prepared from each soil unit, giving 10 series in all. The expected number of IJs in the diluted soil units ranged from 16000 (first dilution) to less than $1 \mathrm{IJ}$ per beaker (last dilution in a series of 16 dilution levels). The mpn was calculated as described below.

\section{Expt 2. Frequency of natural mortality of bait insects in nematode infested and uninfested soil}

Five out of six units of soil were each inoculated and diluted through 14 two-fold dilution levels as described for Expt 1. One unit served as control, inoculated with tap water only, and was also diluted. These data were not used for the calculation of mpn.

The most probable numbers were calculated by (1) testing whether the results of the dilution series conformed to Poisson series by using Moran's test (Moran 1954a,b), and if so, (2) computing the most probable estimate of the numbers of infective units in the undiluted sample. For this purpose a computer program published by Russek \& Colwell (1983) was used and extended to include the Moran test for randomness. This extended program is now obtainable for research purposes by sending a floppy disc to the authors. The Moran test value was calculated and compared with an expected value published by Moran (1954b). The significance levels were obtained from Table 8 of Pearson \& Hartley (1976). Where the data were found to conform to the Poisson series, the mpn of infective units in the undiluted soil was calculated. The extended program calculates the mpn and the associated $95 \%$ confidence 
interval but does not give the confidence limits. The latter were determined as suggested by Cochran (1950).

The data were treated in two ways:

(1) Individual Tenebrio larvae were taken as test units: a positive (parasitised) or negative (living, or dead but not parasitised) score was recorded for each. Each of the seven nematodeinoculated dilution series therefore had 40 replications ( $=$ insects) at each dilution level. Each series was analysed separately.

(2) All 40 Tenebrio larvae in a beaker were treated as one test unit. A positive score was recorded where even a single insect in a beaker was dead. The beakers were then rescored with a positive score being given only where a beaker contained at least one parasitised (rather than dead) insect. Results of seven dilution series were combined, giving seven replicates (= beakers) at each dilution level. The mpn was calculated for the beakers at the most dilute level at which all seven beakers gave a positive reaction. This and all more dilute levels were entered in the calculation procedure. The mpn was calculated by separately using (a) numbers of Tenebrio dead, including Tenebrio which did not die as a result of nematode parasitisation and (b) numbers of Tenebrio dead by nematode parasitisation only. Parasitisation by nematodes was recognised by the characteristic red colour of infected insects; dead insects of other colours were dissected to confirm the absence of nematodes. The data were corrected for natural mortality by Abbott's formula (Abbott, 1925) and this was compared with corrections made by simply subtracting the mean number of Tenebrio dead in the control beakers from the number dead in each beaker of infested soil. Natural Tenebrio mortality in control and Heterorhabditis - inoculated soil was compared by chi-square.

\section{Results}

The results of the baiting procedure from Expt 1 are shown in Table 1. The application of the Moran test to the data based on individual insects for each dilution series separately indicated that parasitisation of the Tenebrio larvae over the dilution series did not follow a Poisson series in any case $(M>2.326, P<0.01)$. It follows that, under the conditions of these experiments, the individual Tenebrio could not be used as the test units for calculating the mpn. In contrast to this, the Moran test on beaker data always gave small values and the test result was never significantly different from a Poisson series at $P=0.01$ (Table 2). These data were therefore judged to conform adequately to the Poisson series. The individual beakers could be used as test units for the calculation of the mpn.

When the proportions of beakers positive (response) were corrected by Abbott's formula (Table 2. column v) and plotted against the log of the expected mean dose ( $\mathrm{m}$ ) of IJs, a curve similar to the dose-response curve obtained for dilution data which follow a Poisson series (e.g. Meynell \& Meynell, 1970) was obtained; in contrast, the curve for Tenebrio mortalities from dilution series 1 (Table 1) is less steep than expected for a Poisson series (Fig. 1). This graphical test supports the results of the Moran test, indicating that the data for beakers were suitable for mpn calculations but the data for insects were not. Both curves lie entirely to the right of the ordinate, over which each would be expected to be centred if all IJs were effective (Meynell \& Meynell, 1970).

The mpn values calculated using beaker data for the dilution level that was expected to have $125 \mathrm{IJs}$ per beaker ranged from 3.7 to 8.9 infective units per beaker for data corrected and not corrected for background mortality, respectively (Table 2). This represents $2.9 \%$ to $7.1 \%$ of the total number of IJs in these infested soil units. Corrections of the insect mortality 
Table 1. Parasitisation of Tenebrio molitor larvae by Heterorhabditis HF85 IJs, in soil of seven independently prepared serial two-fold dilution series (Expt 1). Each soil unit (= beaker) was baited with 40 Tenebrio larvae. The mean natural mortality, calculated from

48 baited control units of soil, was 0.67 dead Tenebrio per beaker

\begin{tabular}{|c|c|c|c|c|c|c|c|}
\hline \multirow{2}{*}{$\begin{array}{l}\text { Expected } \\
\text { number of } \\
\text { IJs per } \\
\text { beaker }\end{array}$} & \multicolumn{7}{|c|}{ Number of insects parasitised } \\
\hline & $\begin{array}{l}\text { Dilution } \\
\text { series } 1\end{array}$ & $\begin{array}{l}\text { Dilution } \\
\text { series } 2\end{array}$ & $\begin{array}{l}\text { Dilution } \\
\text { series } 3\end{array}$ & $\begin{array}{l}\text { Dilution } \\
\text { series } 4\end{array}$ & $\begin{array}{l}\text { Dilution } \\
\text { series } 5\end{array}$ & $\begin{array}{l}\text { Dilution } \\
\text { series } 6\end{array}$ & $\begin{array}{l}\text { Dilution } \\
\text { series } 7\end{array}$ \\
\hline 16000 & 40 & 40 & 40 & 40 & 40 & 40 & 40 \\
\hline 8000 & 32 & 32 & *36 & 37 & 33 & 36 & 34 \\
\hline 4000 & 28 & 32 & 31 & 29 & 30 & $* 25$ & 30 \\
\hline 2000 & 25 & 24 & 26 & $* 26$ & $* 26$ & 26 & 20 \\
\hline 1000 & 20 & 21 & $* * 19$ & 22 & 23 & 14 & $* 17$ \\
\hline 500 & $* 15$ & 18 & 13 & $* 15$ & 11 & *11 & $* 17$ \\
\hline 250 & $* 5$ & $* 8$ & 4 & $* 13$ & $* 8$ & 10 & 4 \\
\hline 125 & 4 & $* * 7$ & 11 & $* 3$ & 6 & 7 & $* * 5$ \\
\hline 62 & 1 & $* 6$ & 2 & 4 & 3 & 2 & 2 \\
\hline 31 & 1 & 4 & 2 & 0 & $* 0$ & 1 & 1 \\
\hline 16 & $* * 0$ & $* * 0$ & 0 & $* * 0$ & $* * * 0$ & 0 & 0 \\
\hline 8 & 2 & 2 & 0 & 1 & $* 0$ & 0 & 0 \\
\hline 4 & 0 & 0 & ${ }^{*} 0$ & $* * 0$ & 0 & 0 & 0 \\
\hline 2 & 0 & 0 & 0 & $*_{0}$ & 0 & $* * 0$ & 0 \\
\hline 1 & $* 0$ & $*_{0}$ & $* 0$ & 0 & 0 & 0 & 0 \\
\hline 0 & 0 & 0 & 0 & 0 & 0 & 0 & 0 \\
\hline $\begin{array}{l}\text { Moran test } \\
(P 0.05=1.645 \\
P 0.01=2.326)\end{array}$ & 4.63 & 7.36 & 4.18 & 4.59 & 4.35 & 4.45 & 3.96 \\
\hline
\end{tabular}

data by either subtraction of control mortalities or the application of Abbott's formula to numbers of Tenebrio dead, had exactly the same outcome in terms of numbers of beakers scoring positive at each level (Table 2 , columns iii $\&$ iv). This showed that the correction procedure based on simple subtraction of the control mortality was as good as the slightly more laborious application of Abbott's formula in this case. In contrast, Abbott's formula made a big impact when it was applied directly to the number of beakers scoring positive (Table 2, column v) and this was an appropriate way to correct these data for background mortality. The confidence limits calculated as suggested by Cochran (1950) agreed well with the confidence interval given by Russek \& Colwell's computer program (Table 2).

The frequency of natural Tenebrio mortality in Expt 1 was significantly higher $(P<0.05)$ in the untreated controls than in the infested soils. A similar result was recorded in Expt 2 with a different batch of HF85 IJs under similar experimental conditions (Table 3). Furthermore, the proportion of beakers showing natural mortality was higher in uninfested than in infested soil in both experiments (Table 3 ).

The infested soil of Expt 1 showed significantly $(P<0.05)$ greater natural mortality of Tenebrio than occurred in the infested soil of Expt 2, at both the Tenebrio level and at the beaker level. In contrast, in the uninfested controls, the differences between the experiments were reversed, though not significantly different, at both Tenebrio or beaker level. 'Natural' insect mortality seemed to be independent of the density of the IJs present, since no significant 
Table 2. Numbers of beakers positive for the criteria listed in columns $(i)$ to $(v)$ from the nine most diluted soils of the dilution series from Expt 1. The most probable number of infective units present in the least diluted beakers has been calculated

\begin{tabular}{|c|c|c|c|c|c|}
\hline & \multicolumn{5}{|c|}{ Number of beakers positive, based on } \\
\hline $\begin{array}{l}\text { Dilution level } \\
\text { (expected number } \\
\text { of }[\mathrm{J} s \text { ) }\end{array}$ & $\frac{i}{\ldots \text { insects dead }}$ & $\begin{array}{c}\text { ii } \\
\ldots \text { insects } \\
\text { parasitised }\end{array}$ & $\begin{array}{l}\text { iii } \\
\text {. Column (i) } \\
\text { corrected for } \\
\text { control } \\
\text { mortality }\end{array}$ & $\begin{array}{l}\text { iv } \\
\text {. Column (i) } \\
\text { corrected by } \\
\text { Abbott's } \\
\text { formula } \\
\text { applied to } \\
\text { insects }\end{array}$ & $\begin{array}{c}\mathrm{v} \\
\text {. Column (i) } \\
\text { corrected by } \\
\text { Abbott's } \\
\text { formula } \\
\text { applied to } \\
\text { beakers }\end{array}$ \\
\hline $\begin{array}{l}1(125) \\
2(62) \\
3(31) \\
4(16) \\
5(8) \\
6(4) \\
7(2) \\
8(1) \\
9(0)\end{array}$ & $\begin{array}{l}7 \\
7 \\
6 \\
4 \\
4 \\
2 \\
2 \\
3 \\
0\end{array}$ & $\begin{array}{l}7 \\
7 \\
5 \\
0 \\
3 \\
0 \\
0 \\
0 \\
0\end{array}$ & $\begin{array}{l}7 \\
6 \\
2 \\
4 \\
2 \\
1 \\
1 \\
0 \\
0\end{array}$ & $\begin{array}{l}7 \\
6 \\
2 \\
4 \\
2 \\
1 \\
1 \\
0 \\
0\end{array}$ & $\begin{array}{l}7 \\
7 \\
5 \\
1 \\
1 \\
0 \\
0 \\
0 \\
0\end{array}$ \\
\hline $\begin{array}{l}\text { Moran test } \\
(P 0.05=1.645 \\
P 0.01=2.326)\end{array}$ & 2.05 & -2.05 & 0.821 & 0.821 & -2.05 \\
\hline $\begin{array}{l}\text { mpn value } \\
95 \% \text { interval } \\
95 \% \text { limits }^{5}\end{array}$ & $\begin{array}{c}8.86 \\
10.07 \\
5.24-14.97\end{array}$ & $\begin{array}{c}4.57 \\
4.88 \\
2.70-7.72\end{array}$ & $\begin{array}{c}3.66 \\
3.89 \\
2.17-6.19\end{array}$ & $\begin{array}{c}3.66 \\
3.89 \\
2.17-6.19\end{array}$ & $\begin{array}{c}4.24 \\
4.52 \\
2.51-7.17\end{array}$ \\
\hline
\end{tabular}

Correction of Tenebrio mortality of each beaker by subtraction of 0.67 Tenebrio (mean natural mortality in control beakers) from the number of dead Tenebrio in each beaker.

Correction of Tenebrio mortality of each beaker by Abbott's formula based on mean natural mortality from control beakers $(0.67$ Tenebrio $=1.67 \%)$.

${ }^{3}$ Number of positive beakers per dilution level corrected by Abbott's formula based on proportion of control beakers containing dead Tenebrio $(=52.1 \%)$.

${ }_{4}^{4}$ Confidence interval calculated as divised by Russek \& Colwell (1983).

'Confidence limits calculated following Cochran (1950).

difference in 'natural' insect mortalities was found in tests comparing the 8 most dilute and 8 least dilute levels of Expt 1 (data of Table 1). These tests examined both the proportion of beakers containing at least one 'naturally' dead insect (Chi-square $=0.401, \mathrm{df}=1, P=0.526$ ) and the proportion of insects 'naturally' dead (Chi-square $=0.025, \mathrm{df}=1, P=0.875$ ).

\section{Discussion}

The mpn calculation could be successfully applied to the experimental data at the beaker level but not at the individual insect level, because of failure to agree with the Poisson series (Moran, 1954a,b). This failure, at the insect level, may be related to differences in susceptibility of the insect hosts or to distribution patterns of either insects or nematodes, since large positive values obtained in the Moran test indicate overdispersion (clumping) in the data. 
Table 3. Frequency of natural mortality of Tenebrio larvae in nematode infested and uninfested soil units. The frequencies were calculated as (a) the number of 'naturally' killed Tenebrio (insects which were apparently dead from causes other than nematodes) as a proportion of the overall number of Tenebrio added to the beakers (n, rows $1 \& 2)$, and (b) the number of beakers showing 'natural' mortalities as a proportion of the total number of beakers ( $n$, rows $3 \& 4)$

\begin{tabular}{|c|c|c|}
\hline & infested soil & uninfested soil \\
\hline $\begin{array}{l}\text { Proportion of insects showing } \\
\text { natural mortality } \\
\text { in Expt } 1\end{array}$ & $\underset{(\mathrm{n}=4480)}{0.92 \%}$ a A & $\begin{array}{l}1.67 \% \quad \text { b A } \\
(\mathbf{n}=1920)\end{array}$ \\
\hline $\begin{array}{l}\text { Proportion of insects showing } \\
\text { natural mortality } \\
\text { in Expt } 2\end{array}$ & $\begin{array}{c}0.18 \% \\
(n=2800)\end{array}$ & $\begin{array}{l}1.79 \% \\
(\mathrm{n}=560)\end{array}$ b A \\
\hline $\begin{array}{l}\text { Proportion of beakers showing } \\
\text { natural mortality } \\
\text { in Expt } 1\end{array}$ & $\begin{array}{l}27.7 \% \\
(\mathrm{n}=112)\end{array}$ a A & $\begin{array}{l}52.1 \% \quad \text { b A } \\
(n=48)\end{array}$ \\
\hline $\begin{array}{l}\text { Proportion of beakers showing } \\
\text { natural mortality } \\
\text { in Expt } 2\end{array}$ & $\begin{array}{c}2.9 \% \\
(\mathrm{n}=70)\end{array}$ & $\begin{array}{l}57.1 \% \quad \text { b A } \\
(\mathrm{n}=14)\end{array}$ \\
\hline
\end{tabular}

Numbers followed by the same letter do not differ significantly $(2 \times 2$ chi-squared, $P<0.05)$

Lower case letters $=$ within a row, upper case letters $=$ within a half column

The flattening of the dose-response curve observed for insect mortalities (Fig. 1) could also be interpreted as an indication of differences in insect susceptibility. When tubes of culture medium differed in suitability for bacterial growth the dose-response curve became flatter than expected from dilution counts with equally suitable tubes (cf. Meynell \& Meyne11, 1970). However, differences in insect susceptibility have less impact on the positive scoring of beakers since one dead insect is enough to give a positive beaker count. Only if there are very few IJs present in the soil and if there are large differences in susceptibility between the insects, will positive beakers be likely to score negative. This was not the case in the experiment (Expt 1) presented, as indicated by the Moran test.

One of the prerequisites for the application of the mpn method is that the organisms to be estimated must be randomly distributed. This means that the IJs have to be randomly distributed between baiting volumes as represented by beakers or insect 'sampling spaces' for mpn assessments at the beaker or insect level, respectively. This situation can be assumed to have existed at the beginning of each assessment when nematodes and insects were thoroughly mixed in the soil, but it is not known for how long the random distribution of nematodes and hosts lasted during the baiting period, despite nematode death or migration. The failure of insect mortality data in the dilution series to conform to a Poisson series could therefore be due to non-random nematode distributions in relation to the individual hosts arising over the 4-day baiting period. In contrast to this, where a whole beaker is taken as one test unit the nematodes are prevented from redistributing themselves with respect to these units by migration (though not by death).

Finally, a third possibility for the departure of Tenebrio data from the Poisson series is suggested: density dependent differences in infective potential or behaviour of the nematodes. Although our knowledge of infective behaviour and strategies of entomopathogenic nematodes in soil is limited, it seems an interesting idea that Heterorhabditis IJs might, depending on their density, act as a group in infecting an insect host. Joint action would also explain why the mpn calculated was so much lower than the number of IJs expected to be 


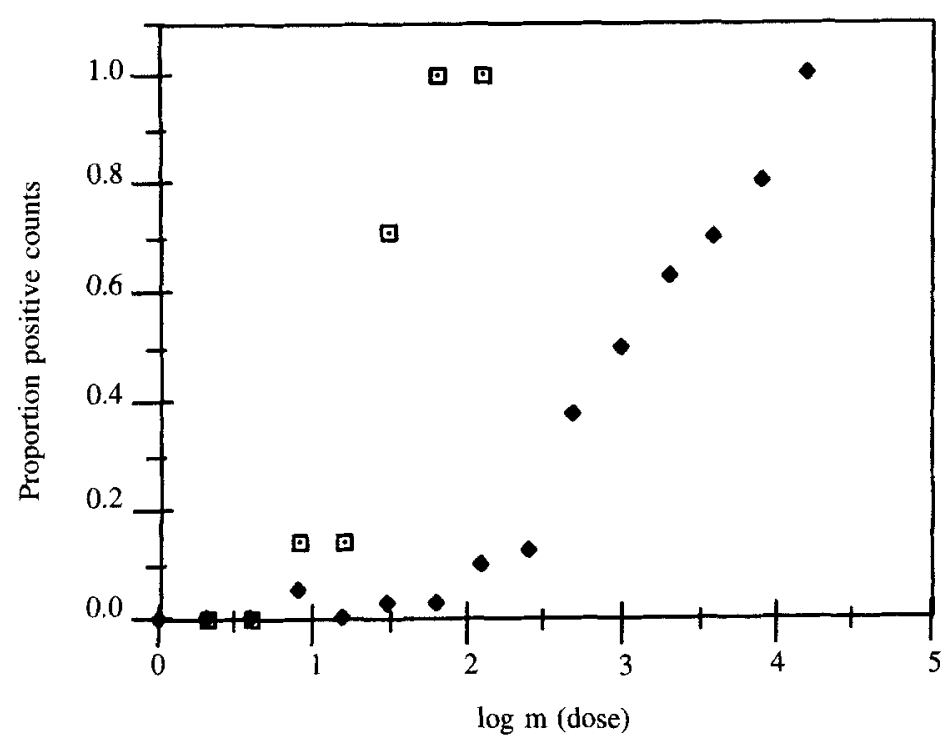

Fig. 1. Dose-response curve for dilution data based on (a) proportion of beakers positive after correction by Abbott's formula (Table 2, column v) and on (b) proportion of Tenebrio parasitised (Table 1, dilution series 1, used as a representative of the seven replicate series) (Expt 1).

a Positive beakers

- Tenebrio mortality

present in a sample. The mpn based on quantal response of the Tenebrio would therefore not give the true density of IJs in the soil samples but the effective density or the quantity of 'infective units'. In cases where more than one organism is required to initiate a reaction, the mpn gives an underestimation of the true density (Cochran, 1950). One possibility is that most of the IJs in the soil are quiescent or otherwise non-infective, and that the infective units each represent a non-quiescent individual $\mathrm{IJ}$. Alternatively, it may well be that, under the almost natural conditions of the present experiment, an insect host like Tenebrio can only be killed by the combined activity of several IJs. Or perhaps several IJs must enter before any can survive inside the insect host during a critical phase where the immune reaction of the insect tries to eliminate the intruders. If there are too few IJs they might even kill the insect by the release of their symbiotic bacteria but not survive themselves. This might be one of the reasons why killed insect hosts occasionally contain the mutualistic bacteria but no living nematodes. Either of these pressures - the need for several IJs to kill the host and the need to ensure successful reproduction - may have resulted in the evolution of a strategy of group invasion. A mechanism by which such group invasion might occur is suggested by the work of Grewal, Gaugler, Kaya \& Wusaty (1993), who found that insects parasitised by steinernematid nematodes were more attractive for conspecific IJs than were unparasitised hosts.

The observed shift of the two dose-response curves in Fig. 1 to the right has also been reported for bacteria when some of the organisms were detected to be 'ineffective' (i.e. to have failed to grow) after inoculation into tubes containing a suitable growth medium (Meynell \& Meynell, 1970). The implication of the shift of the curves also is that not all IJs are infective and/or that the joint action of more than one IJ is required to kill an insect. The shift to the right therefore reinforces those similar conclusions suggested by the disparity between the low mpn values and the expected number of IJs present in the sample. 
Corrections of the insect data for natural mortality, either by the use of Abbott's formula or by subtraction of average control mortalities seems essential, since natural mortalities included in the overall mortality scores may double the mpn value calculated. The lower background mortality in the inoculated than in the uninoculated beakers may be due to some of the insects that were dying naturally in the inoculated beakers being successfully colonised by nematodes. These insects would then have been recorded as dead due to nematode parasitisation. An alternative explanation is that both IJs and 'other causes' are in competition for some highly susceptible, though healthy, insects; IJs are the stronger competitor, thus, where there are IJs present they kill these highly susceptible insects before 'other causes' have a chance. 'Other causes' are then more successful in the absence of nematodes than in the presence. If either of these explanations were true one would expect the proportion of deaths ascribed to background mortality in infested beakers to increase as the density of IJs decreased between dilution levels because the probability of dying insects being invaded should decrease. But a comparison of background mortality in beakers from the first eight dilution levels with beakers from the second eight dilution levels gave no significant difference.

A big advantage of the mpn estimation is that it can be applied even when infectivity is very low because, when only one insect host is killed the whole beaker is scored as positive. By contrast, for an accurate LD50 estimation, for example, it is a requirement that there be mortality responses in the range of about $20-80 \%$ (Wardlaw, 1985). This means that, for the calculation of LD50, relatively large numbers of infective nematodes must be present. Thus, the estimation of mpns could be an alternative to probit analysis, especially when only limited numbers of infective organisms are present. However, a disadvantage of the mpn estimation is the wide confidence intervals, although wide intervals are often also obtained in LD50 calculations. A weakness of the Moran test is that it is unsuitable for the detection of irregular deviations from the normal pattern of results since, for example, the series 5-4-5-2-4-0 and 5-5-4-4-2-0 give the same test value. Also, the test cannot be applied if less than five replicate dilution series are performed. Although the biological significance of the infective unit needs further clarification, mpn seems a useful parameter for use in comparative experiments.

\section{Acknowledgements}

This work was funded by the European Commission under ECLAIR AGRE 0002 and a Trainee Mobility Grant to B Hass. We gratefully acknowledge the statistical advice on most probable numbers given by Mr E Mullins from the Statistical Department, Trinity College, Dublin.

\section{References}

Abbott W S. 1925. A method of computing the effectiveness of an insecticide. Journal of Economic Entomology 18:265-267.

Bedding R A, Akhurst R J. 1975. A simple technique for the detection of insect parasitic rhabditid nematodes in soil. Nematologica 21:109-116.

Cochran W G. 1950. Estimation of bacterial densities by means of the 'most probable number'. Biometrics 6:105-116.

Downes M J. 1972. Counts of infective units of the take-all fungus in Co. Donegal oat soils. Scientific Proceedings of the Royal Dublin Society, Series B, 3:119-126. 
Fan X, Hominick W M. 1991. Efficiency of the Galleria (wax moth) baiting technique for recovering infective stages of entomopathogenic rhabditids (Steinernematidae and Heterorhabditidae) from sand and soil. Revue de Nématolgie 14:381-387.

Gerhardt P, Murray G E, Costilow R N, Nester E W, Wood W A, Krieg N R, Phillips G B. 1981. Manual of methods for general bacteriology. American Society for Microbiology, Washington. 524 pp.

Grewal P S, Gaugler R, Kaya H K, Wusaty M. 1993. Infectivity of the entomopathogenic nematode Steinernema scapterisci (Nematoda: Steinernematidae). Journal of Invertebrate Pathology 62:22-28.

Halvorson H O, Ziegler N R. 1933a. Application of statistics to problems in bacteriology. A means of determining bacterial population by the dilution method. Journal of Bacteriology 25:101-121.

Halvorson H O, Ziegler N R. 1933b. Application of statistics to problems in bacteriology. A consideration of the accuracy of dilution data obtained by using several dilutions. Journal of Bacteriology 26:559-567.

Hass B. 1996. Persistence of entomopathogenic nematodes of the genus Heterorhabditis, Poinar 1975 with emphasis on effects of natural ageing under starvation. Ph.D. Thesis, National University of Ireland. $179 \mathrm{pp}$.

Jones J G. 1979. A guide to methods for estimating microbial numbers and biomass in freshwater. Freshwater Biological Association. Scientific Publication No. 39

Klee A J. 1993. A computer program for the determination of most probable number and its confidence limits. Journal of Microbiological Methods 18:91-98.

Koch A L. 1982. Estimation of the most probable number with a programable pocket calculator. Applied and Environmental Microbiology 43:488-490.

Koppenhöfer A M, Kaya H K, Taormino S P. 1995. Infectivity of entomopathogenic nematodes (Rhabditida: Steinernematidae) at different soil depths and moistures. Journal of Invertebrate Pathology 65:193-199.

McCrady M H. 1915. The numerical interpretation of fermentation tube results. Journal for Infective Diseases 17:183-212.

Meynell G G, Meynell E. 1970. Theory and practice in experimental bacteriology. Cambridge University Press.

Moran P A P. 1954a. The dilution assay of viruses (I). Journal of Hygiene 52:189-193.

Moran P A P. 1954b. The dilution assay of viruses (II). Journal of Hygiene 52:444 446.

Pearson E S, Hartley H O. 1976. Biometrika tables for statisticians, Vol.1. Biometrika Trust, London. $270 \mathrm{pp}$.

Poinar G O. 1975. Description and biology of a new insect parasitic rhabditoid, Heterorhabditis bacteriophora n. gen., n. sp. (Rhabditida; Heterorhabditidae n. fam.). Nematologica 21:463-470.

Rowe R, Todd R, Waide J. 1977. Microtechnique for most probable number analysis. Applied and Environmental Microbiology 33:675-680.

Russek E, Colwell R R. 1983. Computation of most probable numbers. Applied and Environmental Microbiology 45: 1646-1650.

Stevens W L. 1958. Dilution series: a statistical test of technique. Journal of the Royal Statistical Society B 20:205-214.

Taylor J. 1962. The estimation of numbers of bacteria by tenfold dilution series. Journal of Applied Bacteriology 25:54-61.

Turk D, Keyser H H. 1993. Accuracy of most-probable-number estimates of rhizobia for tree legumes. Soil Biology and Chemistry 25:69-74.

Wardlaw A C. 1985. Practical statistics for experimental biologists. John Wiley, New York. 290 pp.

White G F. 1927. A method for obtaining infective nematode larvae from cultures. Science 66:302303.

Woomer P L, Singleton P W, Bohlool B B. 1988. Reliability of the most probable number technique for enumerating rhizobia in tropical soils. Applied and Environmental Microbiology 54:1494-1497.

(Received 31 July 1997) 